\title{
SUSY parameter determination at the LHC using cross sections and kinematic edges
}

\author{
Herbi K. Dreiner, ${ }^{a, b}$ Michael Krämer, ${ }^{c}$ Jonas M. Lindert ${ }^{c}$ and Ben O'Leary ${ }^{c}$ \\ ${ }^{a}$ Bethe Centre for Theoretical Physics 8 Physikalisches Institut, \\ Universität Bonn, D-53115 Bonn, Germany \\ ${ }^{b}$ SCIPP, University of California, \\ Santa Cruz, 95064 CA, U.S.A. \\ ${ }^{c}$ Institut für Theoretische Teilchenphysik und Kosmologie, RWTH Aachen University, \\ D-52056 Aachen, Germany \\ E-mail: dreiner@th.physik.uni-bonn.de, \\ mkraemer@physik.rwth-aachen.de, lindert@physik.rwth-aachen.de, \\ oleary@physik.rwth-aachen.de
}

ABSTRACT: We study the determination of supersymmetric parameters at the LHC from a global fit including cross sections and edges of kinematic distributions. For illustration, we focus on a minimal supergravity scenario and discuss how well it can be constrained at the LHC operating at collision energies of 7 and $14 \mathrm{TeV}$. We find that the inclusion of cross sections greatly improves the accuracy of the SUSY parameter determination, and allows the reliable extraction of model parameters even in the initial phase of LHC data taking with $7 \mathrm{TeV}$ collision energy and $1 \mathrm{fb}^{-1}$ integrated luminosity. Moreover, cross section information may be essential to study more general scenarios, such as those with non-universal gaugino masses, and distinguish them from minimal, universal, models.

Keywords: Supersymmetry Phenomenology

ARXIV EPRINT: 1003.2648 


\section{Contents}

1 Introduction 1

2 Cross sections and cut acceptances 3

2.1 Cross sections $\quad 5$

$\begin{array}{lll}2.2 & \text { Cut acceptances } & 7\end{array}$

$\begin{array}{lll}2.2 .1 & \text { Jet and missing energy cuts } & 7\end{array}$

$\begin{array}{lll}2.2 .2 & \text { Lepton cuts } & 8\end{array}$

$\begin{array}{llr}2.2 .3 & \text { Verification } & 8\end{array}$

3 Numerical results $\quad 9$

$\begin{array}{llr}3.1 & \text { Fit inputs } & 9\end{array}$

$\begin{array}{lll}3.2 & \text { Universal mSUGRA fits } & 13\end{array}$

$\begin{array}{lll}3.3 & \text { Non-universal mSUGRA fits } & 16\end{array}$

4 Conclusions and outlook $\quad 18$

\section{Introduction}

Experiments at the Large Hadron Collider (LHC) will, for the first time, directly explore supersymmetry (SUSY) [1, 2] at the Terascale. Provided that evidence for supersymmetry has been established, a major challenge will be to determine the Lagrangian parameters of the theory, such as the SUSY particle masses, their spins and couplings. These TeV-scale parameters provide essential information on the scheme of supersymmetry breaking and need to be determined with the highest possible accuracy.

A generic LHC signature for supersymmetric models with R-parity conservation $[3,4]$ is that of cascade decays of heavy squarks and gluinos which terminate in the lightest supersymmetric particle (LSP). In many SUSY models the LSP is weakly interacting and provides a viable dark matter candidate $[5,6]$. However, a weakly interacting LSP escapes detection and thus results in missing energy signatures. It is a considerable challenge to reconstruct the sparticle momenta in such cascade decays with missing energy at the LHC and to determine sparticle masses and quantum numbers. The standard technique for analyzing SUSY cascade decays is to consider invariant mass distributions of the finalstate leptons and jets, see e.g. [7-10]. The kinematic endpoints of these distributions are fixed by the masses of the sparticles in the decay chain and yield model-independent information on part of the SUSY mass spectrum. The endpoints can be used as input for global SUSY parameter fits of LHC data [11-16] which determine the high-scale model parameters and thereby test mechanisms of SUSY breaking. (See [17] for a discussion of various fitting methods and tools). However, the endpoints of distributions are only 
sensitive to the kinematics and can not constrain important SUSY parameters such as $\tan \beta$ very well. Moreover, as the endpoints are determined by the difference of masses, there are ambiguities, i.e. points in the SUSY parameter space that have different spectra but similar endpoints. There are further ambiguities from cascade decays with identical final states, but different intermediate particles. It is thus of vital importance to consider additional information for the determination of SUSY parameters at the LHC.

In this paper we address the impact of event rates, i.e. cross sections and branching ratios, on SUSY parameter fits of LHC data. The cross sections are highly sensitive to the masses of the squarks and gluinos that trigger the cascade decays. Branching ratios, on the other hand, depend sensitively on the masses and mixings of SUSY particles further down the decay chain. The inclusion of event rates is technically challenging as the multi-dimensional parameter scan in SUSY fits requires computationally extremely fast and reliable theoretical estimates for cross sections, branching ratios and the effect of experimental cuts. The most straightforward and most flexible approach of estimating event rates from a Monte Carlo simulation for every point in the parameter fit is prohibitively slow [11], so that new strategies need to be developed. Moreover, Monte Carlo calculations are generally based on leading-order perturbation theory, resulting in a substantial theoretical uncertainty in the prediction of event rates. Finally, the statistical fluctuations of Monte Carlo estimates can cause oscillations during the $\chi^{2}$-minimization in gradient based global fits and lead to unstable results [16].

We propose a new approach to include event rates into SUSY parameter fits. Our method is based on a simple parametrization of cross sections and acceptances, ${ }^{1}$ and does not involve Monte Carlo simulations during the $\chi^{2}$-minimization. It is thus fast and reproducible, and it incorporates state-of-the-art higher-order cross section predictions with small theoretical uncertainties. Branching ratios, on the other hand, can be evaluated very quickly with existing computational tools (see e.g. [18]). The information on event rates, i.e. cross sections $\times$ branching ratios $\times$ cut acceptances, has been implemented as an additional observable into Fittino [19] and will become part of the next official release of the Fittino program package [20].

We expect event rate information to be particularly valuable for general SUSY scenarios beyond minimal supergravity, for example those which involve three-body decay modes, so that mass reconstruction via the standard kinematic endpoints is difficult, see e.g. [21, 22]. Moreover, parameter determinations of theories with a general, non-universal, gaugino mass pattern may play a crucial role to distinguish models of supersymmetry breaking [23]. As we shall see, cross sections and branching ratios do not only add information to stabilize a fit with the larger set of parameters in non-universal models, they also allow the determination of specific gaugino masses such as $M_{3}$ much more reliably. Finally, production cross sections are essential to distinguish supersymmetric theories from alternative new physics models, such as universal extra dimensions [24], which predict similar cascade decay signatures at the LHC, but involve new particles with spin quantum numbers differ-

\footnotetext{
${ }^{1}$ We use the terms "acceptance" or "cut acceptance" to denote the fraction of events that passes the experimental selection cuts. Our acceptance estimates are based on parton level calculations and do not yet include detector effects.
} 
ent from the SUSY particle spectrum [25]. A universal extra dimension model could not be distinguished from a supersymmetric theory based on the kinematic information from endpoints of distributions, but would have dramatically different cross sections because of the different particle spins [26-28].

To exemplify the impact of event rates on SUSY parameter fits we have considered the standard SPS1a minimal supergravity (mSUGRA) scenario [29], which has been studied in great detail in previous analyses. SPS1a provides a rich signature at the LHC and can thus be constrained well by standard measurements of kinematic endpoints. It is thus not a scenario where cross sections and branching ratios are expected to have the largest impact. On the other hand, SPS1a allows for a quantitative analysis of the effects of event rates on the parameter fit with realistic error estimates, as discussed in detail in section 3 . Due to the relatively light spectrum it is also one of the SUSY benchmark points that can be studied in the initial phase of the LHC with $7 \mathrm{TeV}$ collision energy and low integrated luminosity. Specifically, we investigate the standard signatures of SPS1a-type models including two or more jets, missing transverse momentum and two leptons of the same flavour but opposite sign. Details of the cuts that specify the signatures will be given in section 2 . We use Fittino to determine the parameters of the SPS1a mSUGRA scenario from the measurement of kinematic edges and event rates at the LHC, considering $7 \mathrm{TeV}$ collision energy with $1 \mathrm{fb}^{-1}$ integrated luminosity and $14 \mathrm{TeV}$ with both $1 \mathrm{fb}^{-1}$ and $10 \mathrm{fb}^{-1}$. As demonstrated in section 3 the inclusion of rates in general stabilizes the fit and significantly improves the error on the mSUGRA parameters, in particular the universal gaugino mass $M_{1 / 2}$ and the ratio of vacuum expectation values $\tan \beta$. Moreover, we find that the inclusion of rates is crucial for the determination of mSUGRA parameters in the initial phase of LHC data taking with $7 \mathrm{TeV}$ collision energy and $1 \mathrm{fb}^{-1}$ integrated luminosity. We also consider a fit where we determine the gaugino mass parameters $M_{1}, M_{2}, M_{3}$ individually at the GUT scale, instead of $M_{1 / 2}$, and show that rate information is important to improve the accuracy of the parameter determination for more general, non-universal, models.

The paper is structured as follows. Section 2 presents details of our method to estimate and parametrize the cross sections and the effects of experimental cuts. The SUSY parameter fits are discussed in section 3 . We conclude in section 4 .

\section{Cross sections and cut acceptances}

The prediction of rates involves the calculation of the production cross section for squarks and gluinos, which dominate the inclusive SUSY signal at hadron colliders, the branching ratios of the supersymmetric particles in the decay chains, and an estimate of the effect of a certain set of experimental cuts. We have considered two powerful and widely studied SUSY signatures [7-10]:

- the inclusive signal of two or more hard jets with each $p_{T \text {,jet }}>50 \mathrm{GeV},|\eta|<2.5$ as well as missing transverse energy of $\mathscr{H}_{T}>100 \mathrm{GeV}$;

- the exclusive signal of two opposite-sign same-flavour leptons $(e$ or $\mu)$, each satisfying $p_{T, \ell}>10 \mathrm{GeV}$ and $|\eta|<2.5$, combined with the above signal of two or more hard jets 


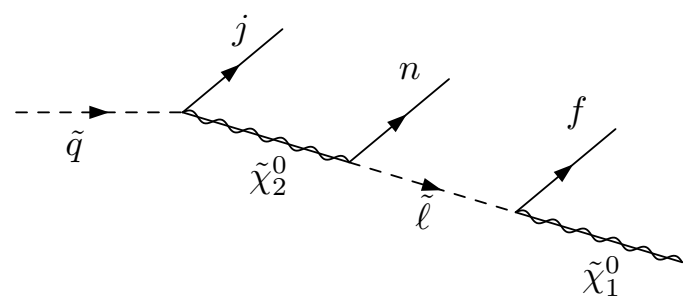

Figure 1. The cascade decay of a squark leading to the leptonic signal. $j$ denotes the quark which should lead to a hard jet, $n$ denotes the near lepton, $f$ denotes the far lepton.

and missing transverse energy. We remove the background from the leptonic decays of tau leptons, charginos and $W^{ \pm}$bosons in the standard way by subtracting events with opposite-sign different-flavour lepton pairs, see [7-10].

Assuming a typical mSUGRA-like mass spectrum with $m_{\tilde{g}}>m_{\tilde{q}}$, the hard jets result from two-body decays of gluinos and squarks, $\tilde{g} \rightarrow q \tilde{q}$ and $\tilde{q} \rightarrow q \tilde{\chi}$, respectively, where $\tilde{\chi}$ denotes a chargino or neutralino, i.e. a model-dependent linear combination of the charged and neutral gauginos and higgsinos. The leptons are produced in chargino/neutralino decays further down the cascade chain, e.g. $\tilde{\chi}_{2}^{0} \rightarrow \ell \tilde{\ell}_{R} \rightarrow \ell \ell \tilde{\chi}_{1}^{0}$, as illustrated in figure 1 . In the SPS1a scenario which we consider for illustration in this paper, the neutralinos $\tilde{\chi}_{3,4}^{0}$ are mainly higgsino, so that the decays $\tilde{q} \rightarrow q \tilde{\chi}_{3,4}^{0}$ are strongly suppressed. Decays into on-shell $Z$ or Higgs, $\tilde{\chi}_{2}^{0} \rightarrow Z \tilde{\chi}_{1}^{0}$ or $\tilde{\chi}_{2}^{0} \rightarrow h \tilde{\chi}_{1}^{0}$, need a sufficient $\tilde{\chi}_{2}^{0}-\tilde{\chi}_{1}^{0}$ mass splitting and are thus not accessible for many mSUGRA scenarios, including SPS1a. Such decays into $Z$ or Higgs will be considered in the future, together with other decay modes with potential significance for more general SUSY models, but are not relevant for the results shown in this paper.

The calculation of the event rates proceeds in various stages. First, for each point in the SUSY parameter space, the mass spectrum and branching ratios are calculated by a spectrum generator. The masses and branching ratios are highly model dependent, but they can be evaluated quickly and can thus be calculated during the $\chi^{2}$-minimization for every point in the parameter space. Our results are based on SPheno [30], which is the default generator used by Fittino. Given the mass hierarchy, we first check which cascade decays can contribute to the signal. The corresponding event rates are proportional to the production cross sections for squarks and gluinos. These cross sections essentially only depend on the squark and gluino masses and on no other SUSY parameters or model assumptions. They can thus be calculated once and for all, including all available higherorder QCD corrections, stored in a $\left(m_{\tilde{q}}, m_{\tilde{g}}\right)$-grid and read out quickly during the fit, see section 2.1. We calculate the cut acceptances using a combination of numerical results stored in look-up tables and analytical calculations of jet energies and lepton energies in the squark rest frame. The calculation of the cut acceptances is less straightforward, and details will be presented in section 2.2. For each contributing cascade, the cross section for the production of the relevant colored sparticles is multiplied by the relevant branching ratios and the cut acceptance, and the total event rate is passed on to Fittino as an observable entering the SUSY parameter fit. 
In the following, we will describe the calculation of cross sections and cut acceptances in some detail.

\subsection{Cross sections}

In supersymmetric models with R-parity conservation, squarks and gluinos are produced in pairs or associated pairs at hadron colliders, $p p \rightarrow \tilde{q} \tilde{q}, \tilde{q} \tilde{q}^{*}, \tilde{q} \tilde{g}, \tilde{g} \tilde{g}+X$. Here we suppress the $\mathrm{SU}(2)$ quantum numbers of the squarks $\tilde{q}=\left(\tilde{q}_{L}, \tilde{q}_{R}\right)$ and do not explicitly state the chargeconjugated processes. The squark and gluino production cross sections are known including next-to-leading order (NLO) SUSY-QCD corrections [31-34], the summation of soft gluon emission [35-39], as well as electroweak contributions and corrections [40-46]. The strength of the SUSY-QCD interactions and thus the production rate is set by the gauge and the Yukawa couplings of the $q q g$ and $q \tilde{q} \tilde{g}$ interactions, respectively. The two couplings are required by supersymmetry to be equal, so that the LO and NLO QCD squark and gluino parton cross sections can be predicted unambiguously in terms of the QCD coupling $g_{\mathrm{s}}$ and the squark and gluino masses, without any further SUSY model dependence. The NLOSUSY QCD corrections have been implemented in the public computer code Prospino [47] and form the basis of our cross section prediction. Note that the strong Yukawa coupling between top quarks, top squarks and Higgs fields gives rise to potentially large mixing effects and mass splitting in the top squark sector. The mixing angle enters the top-squark cross section at NLO, however, the dependence is numerically very weak [34]. Soft gluon resummation leads to a reduction of the scale dependence of the cross section prediction and an enhancement of the NLO QCD cross section for heavy squarks and gluinos with masses $\tilde{m} \gtrsim 1 \mathrm{TeV}$ [39]. These effects will be included in future studies, but are not essential for the results shown in this paper. Electroweak contributions and corrections introduce a dependence on further supersymmetric parameters. Their impact on the inclusive cross sections summed over all squark species is generically small. However, interference effects between the exchange of electroweak gauge bosons and QCD contributions can become large for the production of two SU(2) doublet squarks $p p \rightarrow \tilde{q}_{L} \tilde{q}_{L}^{\prime}[41,42]$. For SPS1a-type scenarios, the electroweak effects are moderate, but they may be larger for more general models, in particular those without gaugino mass unification. Given the residual QCD uncertainties of the production cross section discussed below, we can neglect electroweak contributions for the study of SPS1a presented in this paper. However, such electroweak effects will be addressed in future extensions of this work.

The calculation of the squark and gluino production cross sections involves the calculation of the corresponding parton cross sections, including higher-order SUSY-QCD corrections, and the convolution with parton distribution functions. Numerically, this is a time-consuming task, even at leading order, and would make the inclusion of cross sections in SUSY parameter fits prohibitively slow. Hence, we have calculated the cross sections as a function of $m_{\tilde{q}}$ and $m_{\tilde{g}}$ and stored the results in a grid. As the cross sections are smooth functions of the sparticle masses, the values between grid points can be interpolated reliably. When considering the leptonic signature, we have to distinguish the $\mathrm{SU}(2)$ quantum numbers of the squark, as decays from $\tilde{q}_{R}$ and $\tilde{q}_{L}$ are in general very different. We have thus calculated the LO cross sections for the production of squarks with definite $\mathrm{SU}(2)$ quantum 


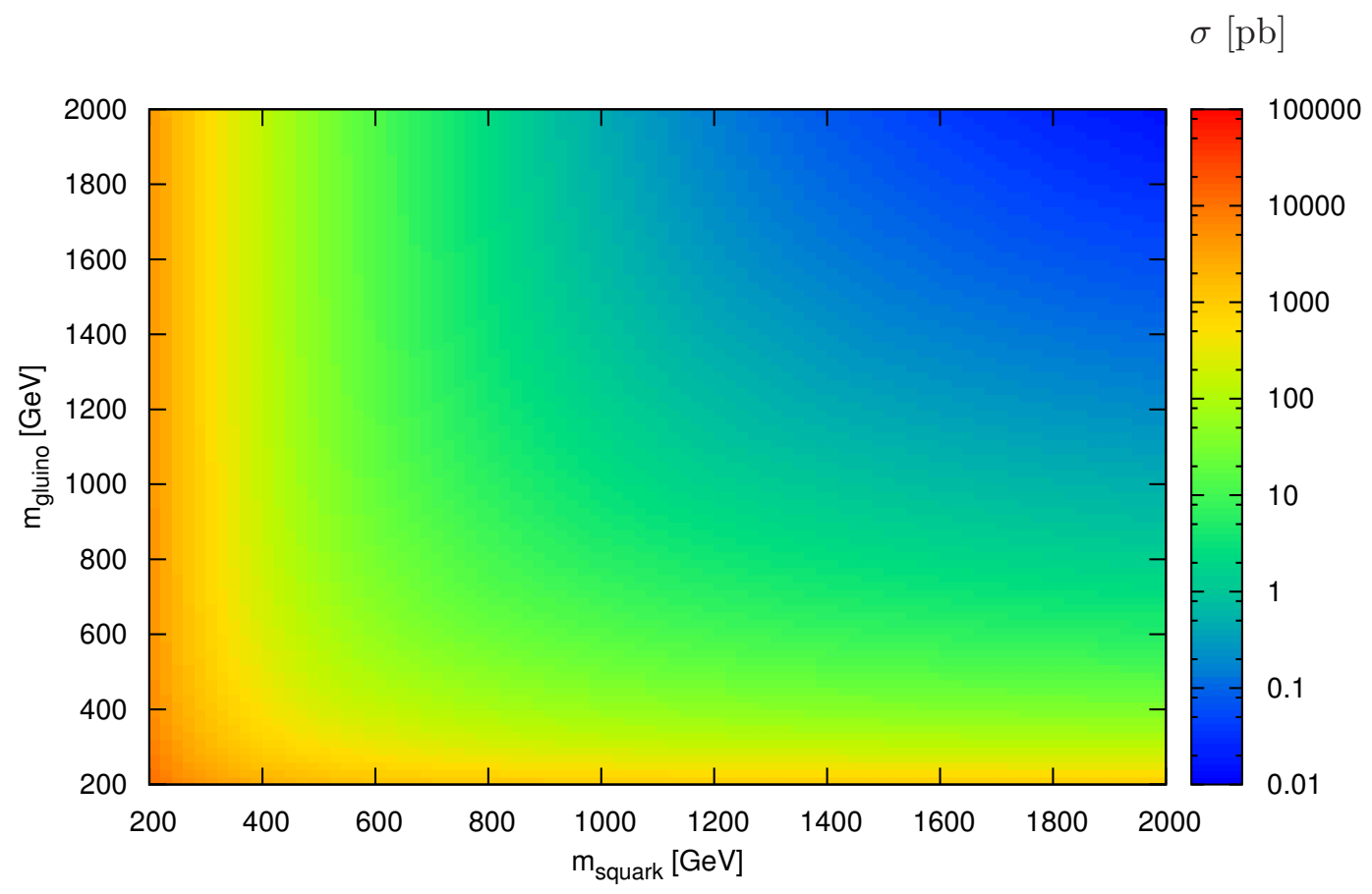

Figure 2. NLO QCD cross section for inclusive squark and gluino production at the LHC (14 TeV) in $\mathrm{pb}$, as a function of the gluino and average squark masses.

number, $\tilde{q}_{L}$ and $\tilde{q}_{R}$, averaging over the masses of $\tilde{q} \in(\tilde{u}, \tilde{d}, \tilde{c}, \tilde{s})$ for each $\mathrm{SU}(2)$ quantum number. Top and, to a lesser extent, bottom squarks mix to form mass eigenstates and are thus treated separately, as mentioned above and explained in detail in ref. [34]. The NLO SUSY-QCD corrections are taken into account through $K$-factors, $K \equiv \sigma_{\mathrm{NLO}} / \sigma_{\mathrm{LO}}$, as provided by Prospino. Note that the calculations [31-33] implemented in Prospino sum over squark SU(2) quantum numbers and do not provide separate $K$-factors for the production of $\tilde{q}_{L}$ and $\tilde{q}_{R}$. This is expected to be a good approximation as the bulk of the NLO corrections results from the pure QCD contributions, in particular soft and collinear gluon emission, which are not sensitive to the SU(2) quantum numbers. Furthermore, we average the $K$-factors for the $\tilde{q} \tilde{q}$ and $\tilde{q} \tilde{q}^{*}$ channels. For our numerical results, we have set the renormalization and factorization scales to the average mass of the produced sparticles and adopted the 2008 MSTW parton distribution functions [48].

To illustrate the sensitivity of the SUSY cross section to the squark and gluino masses, we show in figure 2 the NLO cross sections for $p p \rightarrow \tilde{q} \tilde{q}, \tilde{q} \tilde{q}^{*}, \tilde{q} \tilde{g}, \tilde{g} \tilde{g}+X$ at the LHC with $14 \mathrm{TeV}$ collision energy. In the figure we sum over $\tilde{q} \in(\tilde{u}, \tilde{d}, \tilde{c}, \tilde{s}, \tilde{b})$ and $L / R$ quantum numbers, and average over the squark masses.

Varying $\left(m_{\tilde{q}}, m_{\tilde{g}}\right)$ in the range between $200 \mathrm{GeV}$ and $2 \mathrm{TeV}$, the cross section changes by seven orders of magnitude. It is evident that the sensitivity of the cross section to the sparticle masses should play an important role in SUSY parameter fits.

Let us finally comment on the theoretical error of the cross section prediction. The renormalization and factorization scale uncertainty of the NLO QCD cross section is $\lesssim 10 \%$ 
for squark and gluino masses below approximately $1 \mathrm{TeV}$ [33]. This uncertainty could be reduced further by taking into account soft gluon resummation [39]. In addition, there is the uncertainty due to the parton distribution functions, which, however, is estimated to be below $5 \%$ for sparticle masses less than about $1 \mathrm{TeV}$, see e.g. [48-50]. Taking into account the scale uncertainty, the parton distribution function uncertainty, and other minor uncertainties as described above, we assume an overall theoretical uncertainty of $15 \%$ on our NLO cross section prediction. We shall discuss in section 3.1 how this uncertainty enters the SUSY parameter fit.

\subsection{Cut acceptances}

The possibility of parameterizing the cut acceptances in a generic way hinges upon factorizing the production of the colored sparticles and the subsequent cascade decay. This factorization relies, of course, on the narrow width approximation, which is appropriate as the width of the SUSY particles is in general small, $\Gamma \ll \tilde{m} .^{2}$ We can thus break the problem of estimating cuts for the full production $\otimes$ decay process down to calculating the decay distributions for jets and leptons analytically in the squark rest frame and to numerically estimating the effect of boosting the particles from the squark rest frame to the laboratory frame. The distributions of the squark decay products depend on the SUSY scenario and on many SUSY parameters, but they can be calculated analytically, and thus evaluated quickly during the SUSY parameter scan. The impact of the boost from the squark rest frame to the laboratory frame depends on the dynamics of the production process and is in general difficult to obtain analytically. However, this part can be treated numerically and stored in a look-up table, as it only depends on the production dynamics and thus the squark and gluino masses, and not on any specific details of the SUSY scenario. Our method will be outlined briefly in sections 2.2.1 and 2.2.2; more details can be found in [52]. Currently, the parametrization of cut acceptances is implemented for signals that consist of sequences of two-body decays with the usual mSUGRA mass hierarchy $m_{\tilde{g}}>m_{\tilde{q}}>m_{\tilde{\chi}_{2}^{0}}>m_{\tilde{\ell}}>m_{\tilde{\chi}_{1}^{0}}$. More general scenarios will be considered in future work.

Even though the inclusive cross sections for the production of colored sparticles have large NLO QCD corrections in general, the NLO effects on the differential distributions and thus on the typical boosts of the colored sparticles are small [33]. Hence, for the estimate of the acceptance we rely on leading order calculations.

\subsubsection{Jet and missing energy cuts}

Unfortunately the missing energy cut is very difficult to calculate analytically for a generic decay cascade, and we resorted to a numerical grid of acceptances. The grid has been obtained by a simple parton-level Monte Carlo simulation, decaying all particles by phase space and ignoring spin correlations. This is a legitimate approximation, as we average over charges in the final state. We find that the effects of intermediate decays from the squark to the lightest neutralino tend to average out, and the missing energy cut acceptance is

\footnotetext{
${ }^{2}$ See ref. [51] for new physics scenarios where finite width effects are important; these scenarios are, however, not relevant for the type of models studied in this paper.
} 
approximated well as a function of the hard process (giving the typical squark boosts) and just the mass difference between the squark and the lightest neutralino. The acceptance grid can thus be parametrized by three masses only: $m_{\tilde{g}}, m_{\tilde{q}}$ and $m_{\tilde{\chi}_{1}^{0}}$.

Since all the jets from the decays of the gluinos and squarks were simulated in the process of calculating the missing energy cut acceptances, the jet cut acceptances were also taken from these simulations, though in principle the jet cuts could have been dealt with in the same manner as the lepton cuts described below. It is intended to implement this in the future, to allow for the jet cuts to be specified by the user, rather than hard-coded.

\subsubsection{Lepton cuts}

As argued above, we can estimate the impact of lepton cuts on the production $\otimes$ decay process by calculating the decay distributions to leptons analytically in the squark rest frame and by numerically estimating the effect of the squark boost to the laboratory frame. We have derived analytical expressions for the distributions of near and far leptons in the squark rest frame, which can be evaluated very quickly during the global fit for every point in parameter space. To estimate the lepton acceptance in the laboratory frame, we numerically calculate the acceptance for a generic massless lepton at a given energy in the squark rest frame as a function of the lepton energy and perform a boost to the laboratory frame by means of a simple Monte Carlo calculation. Since the squark is a spinzero particle, the momentum of any one of its final decay products has a flat solid angular distribution in the squark rest frame, though of course there are correlations between the momenta of the decay products. However, we find that the correlations are weak, and that merely multiplying together acceptances for each jet or lepton produces reasonable overall acceptances. The numerical estimate of boosting to the laboratory frame depends on the dynamics of the squark and gluino production process only and is stored in a grid as a function of the squark and gluino masses and the lepton energy. We finally multiply the generic acceptance estimate with the number of leptons at a given energy, as obtained from the analytic calculation of the squark-to-lepton decay. Though the generic acceptances were obtained for a particular choice of transverse momentum cut $(10 \mathrm{GeV})$, they scale simply and can be re-used for different choices of the cut.

\subsubsection{Verification}

To validate our acceptance cut estimates, we performed full parton-level simulations, including spin correlations, with Herwig++ [53] for a set of mSUGRA points chosen randomly with flat priors, restricted then to those points with spectra such that the gluino decayed to on-shell squarks, and that the lightest neutralino was the LSP. The jet and missing energy acceptances from the Herwig++ simulations were compared to those calculated using the methods described above, and were found to agree within $5 \%$ or better. We thus attach an uncertainty to our acceptance cut estimate of $5 \%$. 


\section{Numerical results}

The calculation of the event rates described in section 2 is available in the form of a C++ code and has been incorporated into a currently private modification of Fittino. It will become part of the next official release of the Fittino program library. Fittino is a program which attempts to find the best fit for Lagrangian parameters of supersymmetric models given a set of observables with uncertainties and their covariance matrix. One can choose to perform a fit using simulated annealing or Markov chains (see [16] and references therein), and one can choose to fit to various sets of Lagrangian parameters defined at either the TeV scale or the GUT scale. Since the results presented below were obtained by performing fits with Markov chains to parameters defined at the GUT scale, we briefly outline this process.

Firstly, Fittino reads in its input file, in which all the observables to be used are defined, with their nominal values, uncertainties and correlations. These observables, and the corresponding values adopted in our analysis, are presented in section 3.1. Fittino also reads in the parameters which should be fitted, and a set of starting values for these parameters. Since our aim is to show how the addition of event rates as observables helps to reduce uncertainties on the parameters of the fit, we began the Markov chains at the point which should be found, rather than some other point in the parameter space. At this stage, our code is initialized, and loads the cross section and acceptance grids into memory. Secondly, Fittino begins the process of scanning the parameter space. At each step, it selects a random point near its current point. Then SPheno [30] is used to calculate the TeV-scale spectrum and branching ratios. Fittino uses this spectrum to calculate theory predictions for the observables (either with internal code, including our extension to rates, or by calling external programs). These predictions are then compared to the input values and uncertainties to calculate a likelihood for this new point, which is then used by a Metropolis algorithm [54] to decide whether Fittino moves to this point or rejects it and stays where it is. Finally, the Markov chain is analyzed to calculate a best fit for the parameters and their uncertainties.

\subsection{Fit inputs}

Fittino allows for the inclusion of a wide variety of low-energy and hadron as well as lepton collider observables. For the case study presented here, we restrict ourselves to those observables which are expected to be measured at the LHC. We consider the minimal supergravity SPS1a benchmark point, with parameters $M_{0}=100 \mathrm{GeV}, M_{1 / 2}=250 \mathrm{GeV}$, $A_{0}=-100 \mathrm{GeV}, \tan \beta=10$ and $\operatorname{sign}(\mu)=+1$ [29], corresponding, e.g., to the $\mathrm{TeV}$ scale masses $m_{\tilde{g}}=606 \mathrm{GeV}, m_{\tilde{u}_{L}}=559 \mathrm{GeV}, m_{\tilde{e}_{L}}=177 \mathrm{GeV}, m_{\tilde{\chi}_{2}^{0}}=181 \mathrm{GeV}$ and $m_{\tilde{\chi}_{1}^{0}}=97.1 \mathrm{GeV}$. SPS1a has been studied in great detail in experimental simulations by the ATLAS collaboration [10]. Because of the comparably light spectrum, this benchmark point provides a rich phenomenology, even at low LHC collision energy and luminosity. Furthermore, SPS1a has been adopted as an input for previous SUSY parameter determinations from LHC data $[13,16]$, the results of which may be compared to ours. 
The standard set of LHC observables for SUSY mass determination comprises the endpoints of the four invariant mass distributions that can be constructed from the jet and the two leptons in the cascade depicted in figure 1:

- $m_{\ell \ell}^{\max }$, the dilepton invariant mass edge,

- $m_{q \ell \ell}^{\max }$, the jet-dilepton invariant mass edge,

- $m_{q \ell}^{\text {low }}$, the jet-lepton low invariant mass edge, and

- $m_{q \ell}^{\text {high }}$, the jet-lepton high invariant mass edge.

The definition of these edges and their relation to the sparticle masses in the decay chain can be found in [8]. The $m_{\ell \ell}^{\max }, m_{q \ell \ell}^{\max }, m_{q \ell}^{\text {low }}$ and $m_{q \ell}^{\text {high }}$ endpoints have been analyzed in detail by both the ATLAS and CMS collaborations for different SUSY scenarios [10, 55-58]. At the SPS1a benchmark point, they can be measured at the LHC with a high accuracy of better than $5 \%$, even at a low luminosity of $1 \mathrm{fb}^{-1}[10,16]$. The estimated statistical uncertainties on the measurements of the edges are collected in table 1 for different LHC collision energies and luminosities. We will present results for SUSY parameter fits that involve the four standard edges (labeled group I) and the rates for LHC data analysis at $7 \mathrm{TeV}$ collision energy and $1 \mathrm{fb}^{-1}$ integrated luminosity, and at $14 \mathrm{TeV}$ for both $1 \mathrm{fb}^{-1}$ and $10 \mathrm{fb}^{-1}$.

For $14 \mathrm{TeV}$ collision energy, we also consider a set of observables which involve thirdgeneration particles, the lower endpoint of $m_{q \ell \ell}$ and the stransverse mass [59,60] of the right-handed up squark:

- $m_{q \ell \ell}^{\text {thr. }}$, the jet-dilepton threshold invariant mass edge,

- $m_{T 2}^{\tilde{q}}$, the squark stransverse mass,

- $m_{\tau \tau}^{\max }$, the di-tau invariant mass edge,

- $m_{t b}^{w}$, the weighted top-bottom invariant mass edge, and

- $r_{\tilde{\ell} \tilde{\mathrm{BR}}}$, the ratio of selectron- to stau-mediated $\tilde{\chi}_{2}^{0}$ decays.

This second group of observables (group II) is defined in $[10,16]$ and is much more challenging experimentally than the edges of group I. We are only aware of an ATLAS study $[10,16]$ which quantifies the experimental accuracy to be expected at the LHC operating at $14 \mathrm{TeV}$. In any case, also this second group of measurements may contribute to the SUSY parameter determination at the highest LHC energy and will be included in our analysis below.

The third group (group III), finally, is a set of observables which may only be measurable with at least $10 \mathrm{fb}^{-1}$ of data at $14 \mathrm{TeV}$, and comprises $[10,16]$

- $\Delta m_{\tilde{g} \tilde{\chi}_{1}^{0}}$, the mass difference between the gluino and the LSP,

- $m_{\left(\tilde{\chi}_{4}^{0}\right) \ell \ell}^{\max }$, the dilepton invariant mass edge from the decay of a $\tilde{\chi}_{4}^{0}$, 


\begin{tabular}{|c|c|c|c|c|}
\hline observable & $\begin{array}{l}\text { nominal } \\
\text { value }\end{array}$ & for $7 \mathrm{TeV} / 1 \mathrm{fb}^{-1}$ & $\begin{array}{l}\text { tatistical uncertai } \\
\text { for } 14 \mathrm{TeV} / 1 \mathrm{fb}^{-1}\end{array}$ & for $14 \mathrm{TeV} / 10 \mathrm{fb}^{-1}$ \\
\hline \multicolumn{5}{|l|}{ group I } \\
\hline$m_{\ell \ell}^{\max }$ & 80.4 & 4.4 & 1.5 & 0.43 \\
\hline$m_{q \ell \ell}^{\max }$ & 452.1 & 36.0 & 12.0 & 3.6 \\
\hline$m_{q \ell}^{\text {low }}$ & 318.6 & 19.7 & 6.5 & 3.0 \\
\hline$m_{q \ell}^{\text {high }}$ & 396.0 & 13.5 & 4.5 & 3.9 \\
\hline \multicolumn{5}{|l|}{ group II } \\
\hline$m_{q \ell \ell}^{\text {thr. }}$ & 215.6 & - & 22.8 & 4.1 \\
\hline$m_{T 2}^{q}$ & 531.0 & - & 16.9 & 5.3 \\
\hline$m_{\tau \tau}^{\max }$ & 83.4 & - & 10.8 & 3.4 \\
\hline$m_{t b}^{w}$ & 359.5 & - & 37.0 & 11.7 \\
\hline$r_{\tilde{\ell} \tilde{\tau} \mathrm{BR}}$ & 0.076 & - & 0.008 & 0.003 \\
\hline \multicolumn{5}{|l|}{ group III } \\
\hline$\Delta m_{\tilde{g} \tilde{\chi}_{1}^{0}}$ & 507.7 & - & - & 11.8 \\
\hline$m_{\left(\tilde{\chi}_{4}^{0}\right) \ell \ell}^{\max }$ & 280.6 & - & - & 10.8 \\
\hline$m_{b \ell \ell}^{\text {thr. }}$ & 195.9 & - & - & 17.0 \\
\hline$m_{h}$ & 109.6 & - & - & 1.2 \\
\hline \multirow[t]{2}{*}{ Event rate $[\mathrm{fb}]$} & \multicolumn{2}{|c|}{$7 \mathrm{TeV}$} & \multicolumn{2}{|c|}{$14 \mathrm{TeV}$} \\
\hline & nominal value & uncertainty & nominal value & uncertainty \\
\hline$R_{j j E_{T}}$ & $4.6 \times 10^{3}$ & $9.1 \times 10^{2}$ & $4.8 \times 10^{4}$ & $9.5 \times 10^{3}$ \\
\hline$R_{\ell \ell j j \psi_{T}}$ & $1.6 \times 10^{2}$ & $3.2 \times 10^{1}$ & $1.5 \times 10^{3}$ & $3.0 \times 10^{2}$ \\
\hline
\end{tabular}

Table 1. LHC observables for SPS1a used as inputs to the SUSY parameter fits. The nominal values for masses and branching ratios have been obtained with SPheno. The uncertainty estimates on the observables of groups I, II and III are based on [16] and have been rescaled as described in the main text. Note that the uncertainty estimates for $7 \mathrm{TeV}$ are not based on a detailed experimental simulation but on a simple extrapolation from experimental studies at $14 \mathrm{TeV}$. The event rates include the NLO squark and gluino production cross sections, the branching ratios and the cut acceptances, as described in sections 2.1 and 2.2, respectively. The default overall uncertainty on the rates is assumed to be $20 \%$, see section 2.1 and the main text below. Dimensionful quantities are given in units of $\mathrm{GeV}$ for masses and invariant mass endpoints, or $\mathrm{fb}$ for event rates.

- $m_{b \ell \ell}^{\text {thr. }}$, the $b$-tagged jet-dilepton threshold invariant mass edge, and

- $m_{h}$, the mass of the lightest neutral scalar Higgs boson.

For all SUSY parameter fits we include the two types of event rates defined in section 2 as additional observables:

- $R_{j j E_{T}}$, the inclusive event rate for at least two hard jets with missing transverse energy, and

- $R_{\ell \ell j j \phi_{T}}$, the exclusive event rate for at least two hard jets with missing transverse energy plus a pair of opposite-sign same-flavour light leptons. 


\begin{tabular}{|c|c|c|c|c|}
\hline SPS1a & $\begin{array}{c}M_{0}[\mathrm{GeV}] \\
100\end{array}$ & $\begin{array}{c}M_{1 / 2}[\mathrm{GeV}] \\
250\end{array}$ & $\begin{array}{c}\tan \beta \\
10\end{array}$ & $\begin{array}{c}A_{0}[\mathrm{GeV}] \\
-100\end{array}$ \\
\hline \multicolumn{5}{|l|}{$7 \mathrm{TeV}$ and $1 \mathrm{fb}^{-1}$} \\
\hline $\mathrm{I}+\operatorname{rates}_{\Delta=100 \%}$ & $101.7_{-13.0}^{+7.4}$ & $247.8_{-8.5}^{+17.2}$ & $8.9_{-5.2}^{+16.1}$ & $-227.9_{-311}^{+2045}$ \\
\hline $\mathrm{I}+\operatorname{rates}_{\Delta=50 \%}$ & $101.9_{-12.9}^{+6.9}$ & $248.5_{-7.1}^{+12.5}$ & $8.3_{-3.7}^{+8.9}$ & $-237.6_{-237}^{+2070}$ \\
\hline$I+\operatorname{rates}_{\Delta=20 \%}$ & $99.0_{-9.1}^{+9.9}$ & $250.0_{-6.5}^{+8.7}$ & $10.7_{-8.8}^{+4.0}$ & $55.2_{-254}^{+1048}$ \\
\hline \multicolumn{5}{|l|}{$14 \mathrm{TeV}$ and $1 \mathrm{fb}^{-1}$} \\
\hline $\mathrm{I}+\operatorname{rates}_{\Delta=20 \%}$ & $99.7_{-5.7}^{+4.3}$ & $251.1_{-5.8}^{+7.5}$ & $11.2_{-5.1}^{+3.5}$ & $-50.9_{-350}^{+1233}$ \\
\hline $\mathrm{I}+\mathrm{II}$, rates & $99.8_{-4.4}^{+3.3}$ & $249.7_{-5.2}^{+6.6}$ & $10.1_{-3.2}^{+3.8}$ & $-94.1_{-216}^{+1610}$ \\
\hline $\mathrm{I}+\mathrm{II}+\operatorname{rates}_{\Delta=20 \%}$ & $99.8_{-4.2}^{+3.9}$ & $251.3_{-5.0}^{+5.0}$ & $10.7_{-3.1}^{+3.1}$ & $-55.7_{-233}^{+263}$ \\
\hline \multicolumn{5}{|l|}{$14 \mathrm{TeV}$ and $10 \mathrm{fb}^{-1}$} \\
\hline$I+\operatorname{rates}_{\Delta=20 \%}$ & $100.0_{-3.2}^{+2.9}$ & $250.7_{-3.0}^{+2.9}$ & $11.0_{-3.1}^{+2.5}$ & $-63.3_{-192}^{+165}$ \\
\hline $\mathrm{I}+\mathrm{II}$, rates & $100.1_{-1.9}^{+1.7}$ & $250.4_{-1.7}^{+1.2}$ & $10.1_{-1.0}^{+1.1}$ & $-89.8_{-80.3}^{+70.4}$ \\
\hline $\mathrm{I}+\mathrm{II}+\operatorname{rates}_{\Delta=20 \%}$ & $100.3_{-1.9}^{+1.6}$ & $250.4_{-1.6}^{+1.4}$ & $10.2_{-1.0}^{+1.2}$ & $-96.5_{-68.5}^{+86.3}$ \\
\hline $\mathrm{I}+\mathrm{II}+\mathrm{III}$, rates & $100.2_{-1.6}^{+1.4}$ & $250.3_{-1.4}^{+1.1}$ & $10.1_{-0.8}^{+0.8}$ & $-94.6_{-55.0}^{+48.2}$ \\
\hline $\mathrm{I}+\mathrm{II}+\mathrm{III}+$ rates $_{\Delta=20 \%}$ & $100.1_{-1.5}^{+1.6}$ & $250.3_{-1.4}^{+1.1}$ & $10.3_{-1.0}^{+0.7}$ & $-90.3_{-57.7}^{+52.1}$ \\
\hline
\end{tabular}

Table 2. Fits to universal mSUGRA parameters for SPS1a, with ("+rates") and without ("rates") event rates as an observable. The symbols I, II and III refer to the inclusion of the groups of previously considered observables (mainly edges) defined in the main text. For $7 \mathrm{TeV}$ and $1 \mathrm{fb}^{-1}$ we show results based on $100 \%, 50 \%$ and $20 \%$ error on rates. For the analyses at $14 \mathrm{TeV}$ we always assume a rate uncertainty of $20 \%$.

The theoretical uncertainty of our rate prediction includes $15 \%$ uncertainty on the NLO calculation of the squark and gluino cross section and $5 \%$ uncertainty on our acceptance estimate. Further theoretical uncertainties arise from the renormalization group running [61], the conversion of Lagrangian parameters into physical masses [62] and the calculation of the branching ratios, all obtained at finite order in perturbation theory. These effects are, however, estimated to be small compared to the $15 \%$ production cross section uncertainty and can safely be neglected. For SPS1a, the statistical uncertainty on the rate is also significantly smaller than the theory uncertainty: even at $7 \mathrm{TeV}$ collision energy with $1 \mathrm{fb}^{-1}$ integrated luminosity, we expect about 150 signal events for the exclusive signature including two opposite-sign same-flavour leptons. We may thus simply assume an overall rate uncertainty of $20 \%$ as a default for our analysis of SPS1a. Note, however, that prior to data it is difficult to reliably estimate the systematic uncertainty of a rate measurement. Therefore, for the initial phase of LHC data analysis at $7 \mathrm{TeV}$ collision energy and with $1 \mathrm{fb}^{-1}$ integrated luminosity, we show parameter fits based on $20 \%, 50 \%$ and $100 \%$ rate uncertainty. For the analysis of data taken in the advanced stage of the LHC at $14 \mathrm{TeV}$ we assume that systematic errors of rate measurements are well under control and adopt a rate uncertainty of $20 \%$.

All observables are collected in table 1 together with their estimated statistical uncertainties. The overall experimental uncertainties that enter the fit also include a systematic 
I, rates

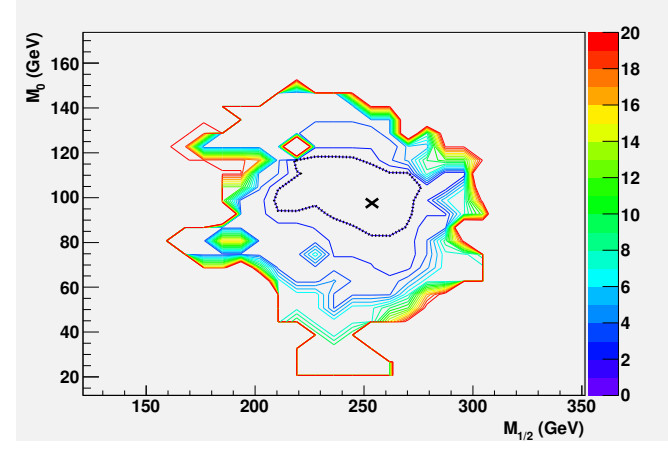

$\mathrm{I}+\operatorname{rates}_{\Delta=50 \%}$

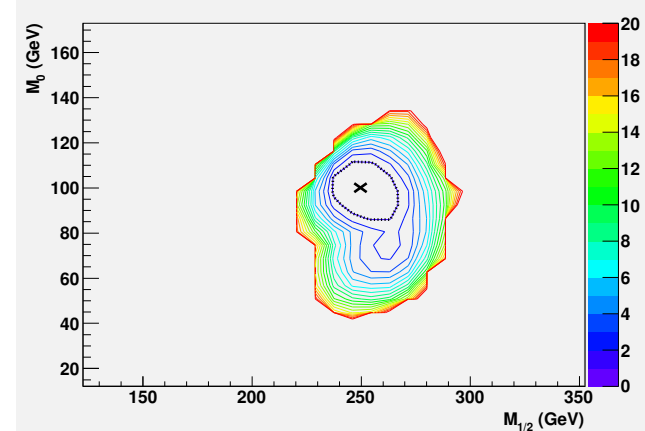

$\mathrm{I}+\operatorname{rates}_{\Delta=100 \%}$

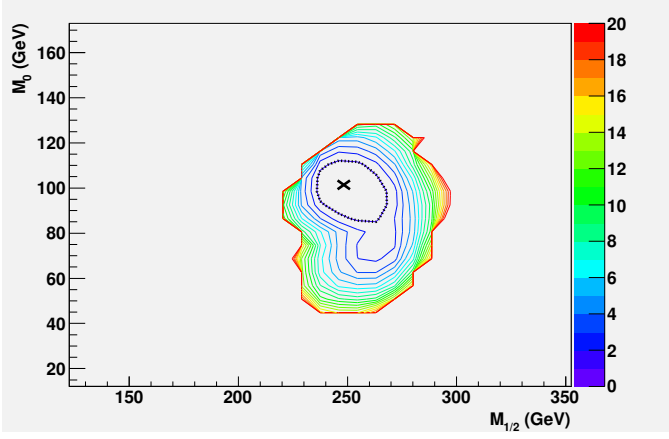

$\mathrm{I}+\operatorname{rates}_{\Delta=20 \%}$

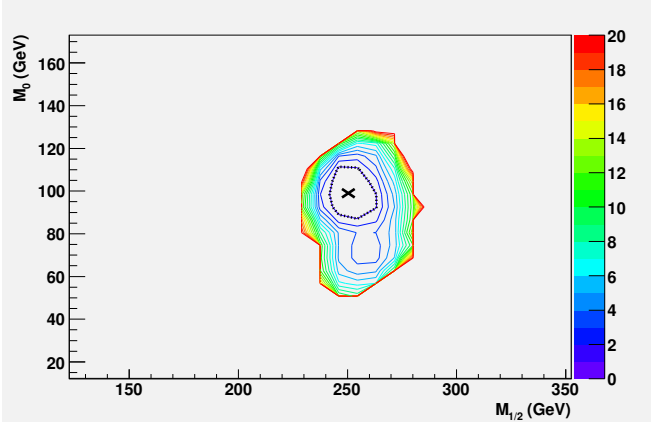

Figure 3. $\Delta \chi^{2}=-2 \ln \mathcal{L}+2 \ln \mathcal{L}_{\max }$ contours showing $M_{0}$ against $M_{1 / 2}$ for $7 \mathrm{TeV} / 1 \mathrm{fb}^{-1}$ data. Fits are based on the four standard edges of group I without rates ("I, rates", upper left) and with rates ("I + rates"), assuming $100 \%, 50 \%$ and $20 \%$ rate uncertainty. $\mathcal{L}$ is the two-dimensional profile likelihood and $\mathcal{L}_{\text {max }}$ the global maximum of the likelihood. The black dotted contours represent $\Delta \chi^{2}=1$ contours. See [16] for more details.

uncertainty given by the jet and lepton energy scale, which is assumed to be $5 \%$ (1\%) and $0.2 \%(0.1 \%)$, respectively, for $1(10) \mathrm{fb}^{-1}[10,16]$. The uncertainties on the endpoints related to the lepton and jet energy scale are considered $100 \%$ correlated among different measurements. Further systematic uncertainties for some of the observables of groups II and III can be found in [16]. The statistical uncertainties in table 1 are estimated by rescaling the uncertainties for $14 \mathrm{TeV}$ collision energy listed in [16] by $\sqrt{R_{N}}$, where $R_{N}$ is the ratio of expected numbers of events passing appropriate cuts when going from LO at $14 \mathrm{TeV}$ (on which the simulations in [16] are based) to NLO at $14 \mathrm{TeV}$ or NLO at $7 \mathrm{TeV}$.

Our fits are obtained from Markov chains with $10^{5}$ steps. The frequentist interpretation of these Markov chains is used to calculate the errors of the parameters, see [16] for details. Including rates slows down the computation by only about $30 \%$; further optimization is possible and will be implemented in the public release of the code.

\subsection{Universal mSUGRA fits}

In this section and section 3.3 below we present the main results of this paper and demonstrate that rates significantly improve the fits of SUSY parameters at the LHC. We first 


\section{I + II, rates}

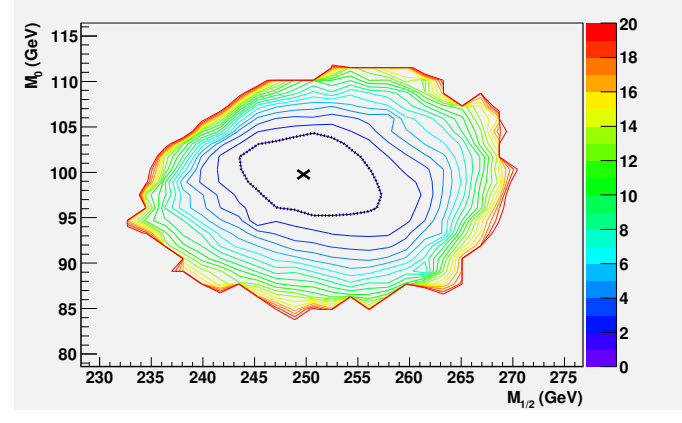

$\mathrm{I}+\operatorname{rates}_{\Delta=20 \%}$

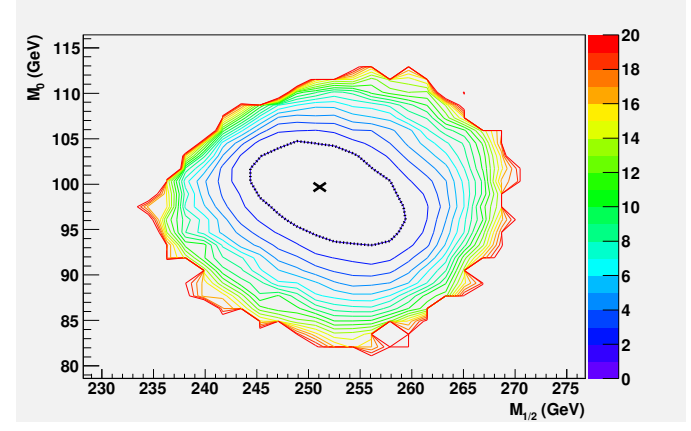

$\mathrm{I}+\mathrm{II}+\operatorname{rates}_{\Delta=20 \%}$

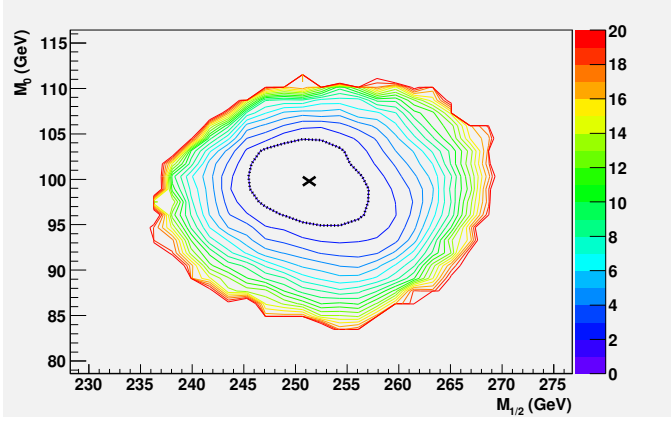

Figure 4. $\Delta \chi^{2}$ contours showing $M_{0}$ against $M_{1 / 2}$ for $14 \mathrm{TeV} / 1 \mathrm{fb}^{-1}$ data. Fits are based on the four standard edges of group I with rates (upper right), and on the observables of groups I and II with (lower right) and without rates (lower left).

discuss the determination of the universal mSUGRA parameters $M_{0}, M_{1 / 2}, \tan \beta$ and $A_{0}$ (with $\operatorname{sign}(\mu)=+1$ fixed) for the SPS1a benchmark scenario. We show results for $7 \mathrm{TeV}$ LHC collision energy with $1 \mathrm{fb}^{-1}$ luminosity, and $14 \mathrm{TeV}$ with both $1 \mathrm{fb}^{-1}$ and $10 \mathrm{fb}^{-1}$.

Let us first consider LHC data taken at $7 \mathrm{TeV}$ and $1 \mathrm{fb}^{-1}$. As mentioned above, it is not clear which observables beyond the four standard edges can be measured with what accuracy during such an initial phase of LHC data analysis. We thus address the question of what information can be obtained from the four edges and the event rates only. The results of our fit are collected in table 2. Remarkably, we find that we can constrain the universal scalar and gaugino masses with an error of $10 \%$ and $3 \%$, respectively, if the overall uncertainty on rates is around $20 \%$. Even with $100 \%$ rate uncertainty, we can determine $M_{0}$ and $M_{1 / 2}$ with an accuracy of $10 \%$. Moreover, with a $20 \%$ measurement of rates, the fit reproduces the correct value of $\tan \beta$ with a reasonable error. Note that the tri-linear coupling $A_{0}$ remains essentially unconstrained: while $M_{0}, M_{1 / 2}$ and $\tan \beta$ are directly sensitive to masses and cross sections $\times$ branching ratios, the tri-linear coupling enters the fit only much more indirectly through mixing in the third generation. We emphasize that the impact of the rate information is crucial: including the four edges of group I alone leads to very large errors and an unreliable fit, as one can judge from the profile likelihood contours shown in figure 3. Even adding rates with a large uncertainty of $100 \%$ leads to a significant improvement of the parameter determination, see upper right plot in figure 3 . 


\section{I + II, rates}

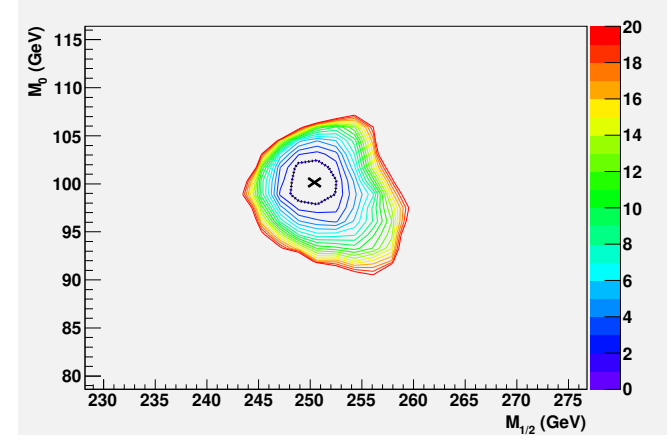

I + II + III, rates

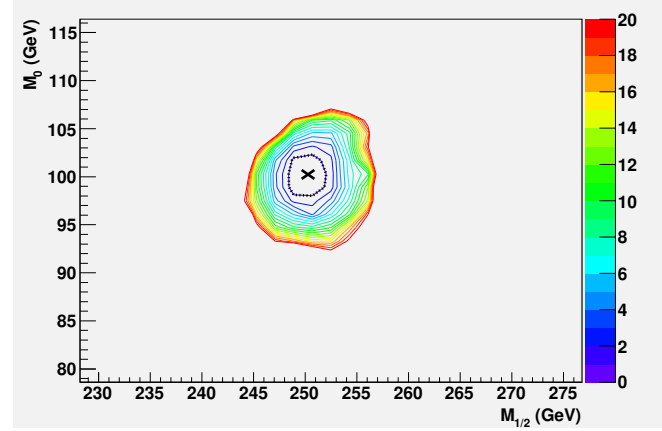

$\mathrm{I}+\operatorname{rates}_{\Delta=20 \%}$

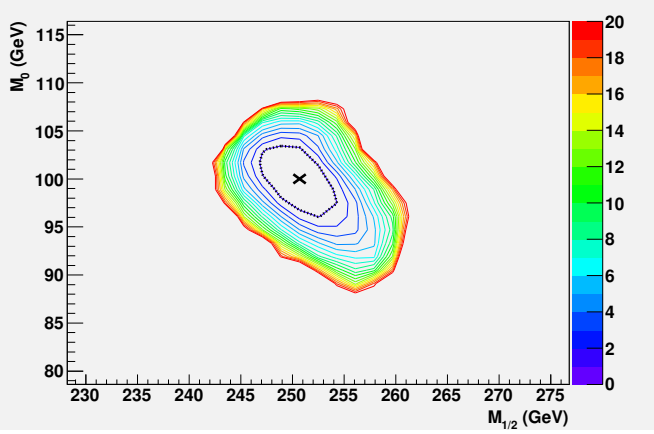

$$
\mathrm{I}+\mathrm{II}+\operatorname{rates}_{\Delta=20 \%}
$$

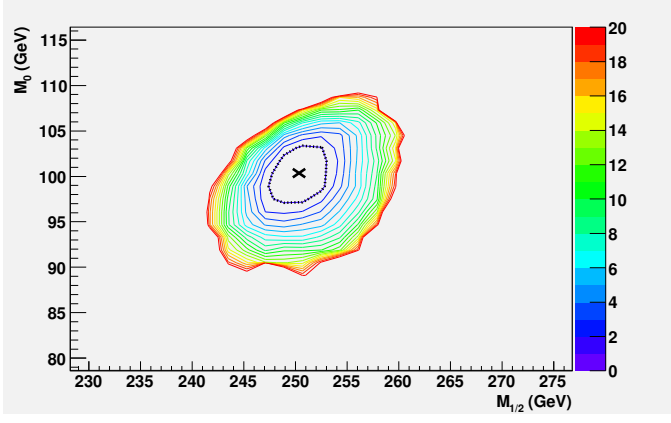

$$
\mathrm{I}+\mathrm{II}+\mathrm{III}+\operatorname{rates}_{\Delta=20 \%}
$$

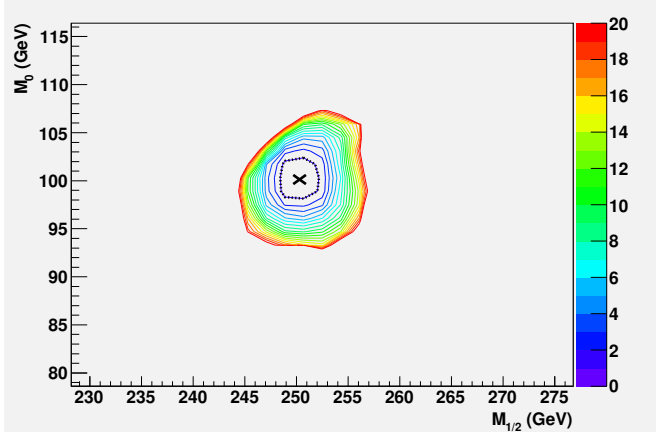

Figure 5. $\Delta \chi^{2}$ contours showing $M_{0}$ against $M_{1 / 2}$ for $14 \mathrm{TeV} / 10 \mathrm{fb}^{-1}$ data. Fits are based on the four standard edges of group I with rates (upper right), on the observables of groups I and II with (middle right) and without rates (middle left), and on observables of groups I, II, and III with (lower right) and without rates (lower left).

(Note that we needed 2 million Markov chain steps in total to derive the plot showing the fit without rates, while the fits with rates have been obtained with $10^{5}$ steps only.)

At $14 \mathrm{TeV}$ collision energy and $1 \mathrm{fb}^{-1}$ luminosity, the statistical uncertainty on the measurement of edges is considerably reduced, see table 1 . We thus obtain a better determination of the mSUGRA parameters with rates and the basic edges of group I, in particular for the common scalar mass $M_{0}$. Note that the errors on $\tan \beta$ and $M_{1 / 2}$ in the fit "I + rates" in table 2 would be twice as large had we adopted a $100 \%$ rate uncertainty. 


\begin{tabular}{|c|c|c|c|c|c|c|}
\hline SPS1a & $\begin{array}{c}M_{0}[\mathrm{GeV}] \\
100\end{array}$ & $\begin{array}{c}M_{1}[\mathrm{GeV}] \\
250\end{array}$ & $\begin{array}{c}M_{2}[\mathrm{GeV}] \\
250\end{array}$ & $\begin{array}{c}M_{3}[\mathrm{GeV}] \\
250\end{array}$ & $\begin{array}{c}\tan \beta \\
10\end{array}$ & $\begin{array}{c}A_{0}[\mathrm{GeV}] \\
-100\end{array}$ \\
\hline \multicolumn{7}{|l|}{$7 \mathrm{TeV}$ and $1 \mathrm{fb}^{-1}$} \\
\hline $\mathrm{I}+\operatorname{rates}_{\Delta=100 \%}$ & $97.0_{-41.2}^{+64.3}$ & $242.3_{-91.5}^{+173.7}$ & $241.8_{-48.3}^{+186.0}$ & $249.3_{-19.6}^{+68.5}$ & $5.0 \stackrel{+10.0}{+2.0}$ & $-446.2 \stackrel{+1532}{-195.6}$ \\
\hline $\mathrm{I}+\operatorname{rates}_{\Delta=50 \%}$ & $103.1+27.0$ & $248.9 \stackrel{+107.7}{+12.4}$ & $250.0_{-46.1}^{+72.9}$ & $245.5_{-9.8}^{+32.9}$ & $7.8_{-4.2}^{+11.8}$ & $-269.9 \stackrel{+1247}{-261.0}$ \\
\hline $\mathrm{I}+\operatorname{rates}_{\Delta=20 \%}$ & $91.1_{-36.1}^{+27.3}$ & $236.5 \begin{array}{l}+67.1 \\
-57.9\end{array}$ & $242.6_{-33.7}^{+51.6}$ & $251.0_{-8.5}^{+9.5}$ & $10.5_{-7.3}^{+7.4}$ & $-6.0 \begin{array}{l}+1088 \\
-582\end{array}$ \\
\hline \multicolumn{7}{|l|}{$14 \mathrm{TeV}$ and $1 \mathrm{fb}^{-1}$} \\
\hline $\mathrm{I}+$ rates $_{\Delta=20 \%}$ & $98.5 \stackrel{+16.5}{-18.4}$ & $245.8_{-40.7}^{+55.7}$ & $244.2_{-19.4}^{+42.1}$ & $250.3 \stackrel{+11.1}{+7.0}$ & $6.2_{-2.0}^{+11.0}$ & $-389.9 \stackrel{+2195}{-169}$ \\
\hline $\mathrm{I}+\mathrm{II}$, rates & $102.7_{-21.4}^{+9.4}$ & $258.0+32.5$ & $255.4+43.6$ & $251.4+\frac{1.9}{+9.2}$ & $10.3_{-3.0}^{+5.9}$ & $-102.0 \begin{array}{l}+1377 \\
-186\end{array}$ \\
\hline $\mathrm{I}+\mathrm{II}+$ rates $_{\Delta=20 \%}$ & $98.6_{-11.2}^{+12.6}$ & $249.6_{-24.7}^{+31.7}$ & $248.7_{-15.5}^{+24.9}$ & $252.1_{-7.1}^{+6.0}$ & $9.5_{-2.7}^{+6.6}$ & $-127.5_{-204}^{+790}$ \\
\hline \multicolumn{7}{|l|}{$14 \mathrm{TeV}$ and $10 \mathrm{fb}^{-1}$} \\
\hline $\mathrm{I}+\operatorname{rates}_{\Delta=20 \%}$ & $99.2_{-9.3}^{+13.3}$ & $253.7_{-30.5}^{+37.1}$ & $256.9_{-23.9}^{+20.2}$ & $250.9_{-5.7}^{+8.6}$ & $14.3_{-9.7}^{+1.7}$ & $186.6 \stackrel{+239}{+761}$ \\
\hline $\mathrm{I}+\mathrm{II}$, rates & $\begin{array}{r}98.4+7.9 \\
-4.1\end{array}$ & $246.8_{-13.0}^{+22.5}$ & $248.0 \begin{array}{l}+12.6 \\
-6.8\end{array}$ & $249.2_{-3.2}^{+5.2}$ & $9.6_{-0.8}^{+1.7}$ & $-117.5+83.1$ \\
\hline $\mathrm{I}+\mathrm{II}+\operatorname{rates}_{\Delta=20 \%}$ & $102.0_{-5.7}^{+2.5}$ & $258.3_{-20.0}^{+9.2}$ & $254.3_{-10.2}^{+6.0}$ & $251.9+2.1$ & $9.9+1.4$ & $-124.7 \stackrel{+101}{-63.6}$ \\
\hline $\mathrm{I}+\mathrm{II}+\mathrm{III}$, rates & $99.1+5.0$ & $245.9+19.5$ & $248.4{ }_{-5.2}^{+10.3}$ & $248.7+5.2$ & $9.9+0.9$ & $-98.6_{-48.0}^{+41.7}$ \\
\hline $\mathrm{I}+\mathrm{II}+\mathrm{III}+\operatorname{rates}_{\Delta=20 \%}$ & $99.1_{-3.5}^{+6.4}$ & $251.3_{-11.2}^{+0.5}$ & $250.4{ }_{-5.1}^{+9.9}$ & $251.2_{-4.6}^{+3.0}$ & $9.9+0.7$ & $-101.0+47.9$ \\
\hline
\end{tabular}

Table 3. Fits to mSUGRA parameters with non-universal gaugino masses for SPS1a, with ("+rates") and without ("rates") event rates as an observable. I, II and III refer to the inclusion of the groups of previously considered observables (mainly edges) defined in the main text. For $7 \mathrm{TeV}$ and $1 \mathrm{fb}^{-1}$ we show results based on $100 \%, 50 \%$ and $20 \%$ error on rates. For the analyses at $14 \mathrm{TeV}$ we always assume a rate uncertainty of $20 \%$.

Adding the observables of group II, which involve information on third-generation particles, the lower endpoint of $m_{q \ell \ell}$ and the stransverse mass, does not lead to a significant improvement. The rate information together with the standard edges thus seems sufficient to determine the parameters of an mSUGRA fit to SPS1a-type SUSY scenarios in the initial phase of a high energy LHC run. Our results for $14 \mathrm{TeV}$ and $1 \mathrm{fb}^{-1}$ without rates, presented in table 2 and figure 4, are consistent with the results presented in ref. [16], specifically table 15 thereof.

At $14 \mathrm{TeV}$ and $10 \mathrm{fb}^{-1}$ also the systematical errors from lepton and jet energy scales are expected to improve, leading to a further reduction of the error on edges. Fitting the four edges of group I and the rates leads to a very accurate determination of the mSUGRA parameters $M_{0}$ and $M_{1 / 2}$ and of $\tan \beta$. At high energy and high luminosity, the observables of group III may become accessible. With all 13 observables of groups I, II and III included, the fit to the SPS1a mSUGRA scenario is so well constrained that adding rates does not lead to a significant improvement, see figure 5. Our results are consistent with those presented in table 16 of [16].

\subsection{Non-universal mSUGRA fits}

Let us now briefly discuss a more general class of fits where we attempt to determine the GUT-scale gaugino mass parameters $M_{1}, M_{2}$ and $M_{3}$ as fit parameters individually, instead of a common mass $M_{1 / 2}$. Models without gaugino mass unification at the GUT scale can lead to very different phenomenology, see e.g. [63-65], and have, to our knowledge, so far not been considered in SUSY parameter fits of LHC data alone. As in section 3.2, we 
I, rates

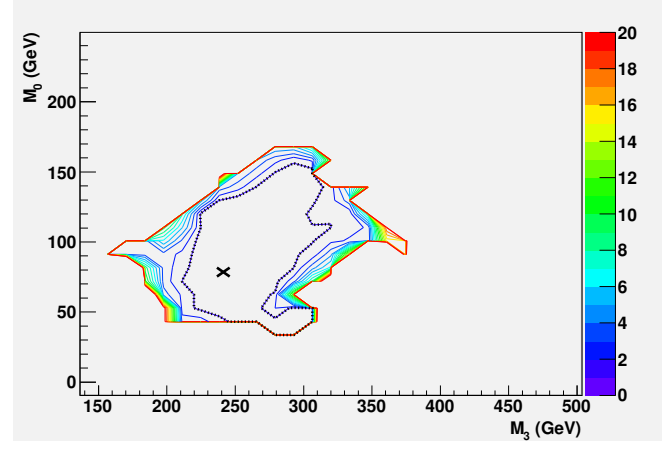

$\mathrm{I}+\operatorname{rates}_{\Delta=50 \%}$

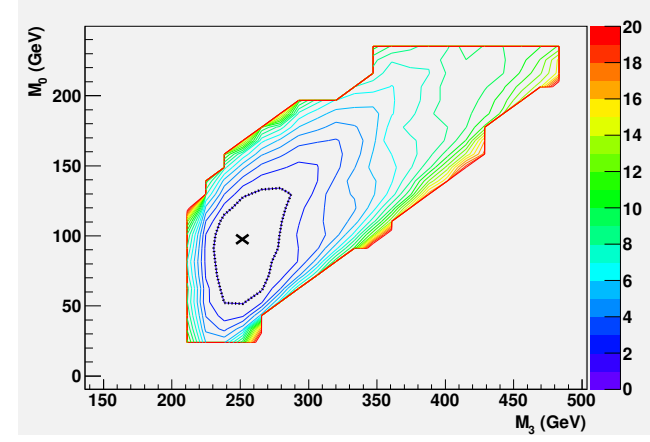

$\mathrm{I}+\operatorname{rates}_{\Delta=100 \%}$

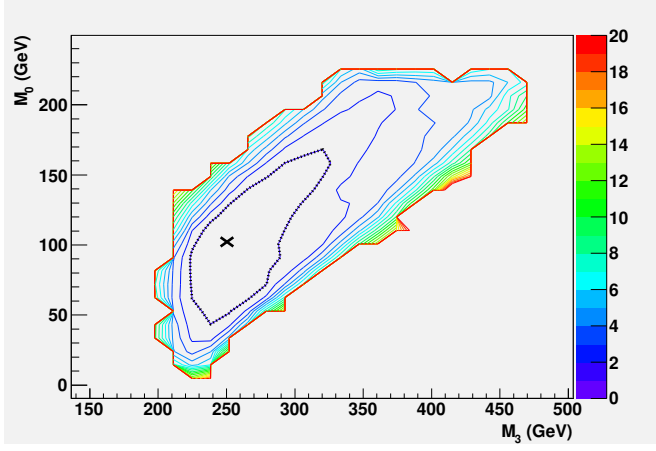

$\mathrm{I}+\operatorname{rates}_{\Delta=20 \%}$

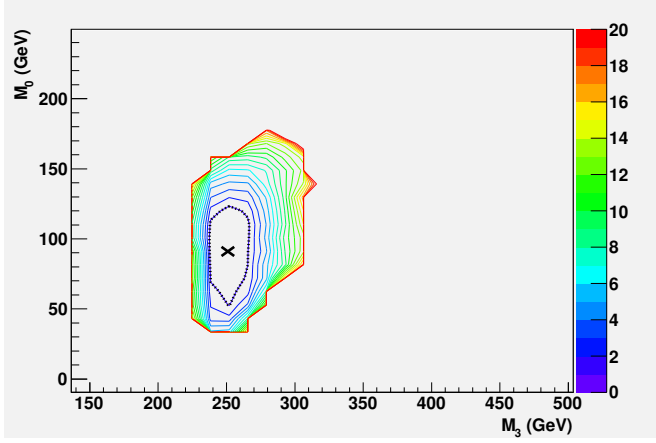

Figure 6. $\Delta \chi^{2}$ contours showing $M_{0}$ against $M_{3}$ for $7 \mathrm{TeV} / 1 \mathrm{fb}^{-1}$ data. Fits are based on the four standard edges of group I without (upper left) and with rates, assuming $100 \%, 50 \%$ and $20 \%$ rate uncertainty.

use the SPS1a observables collected in table 1 as input. Even though SPS1a is defined by $M_{1}=M_{2}=M_{3}=250 \mathrm{GeV}$ at the GUT scale, our results presented below demonstrate the importance of rates for the determination of individual gaugino mass parameters, crucial for the analysis of more general, non-universal, models.

Analogous to what we observe for the fit of universal mSUGRA parameters, we find that a measurement of the four standard edges of group I alone is not sufficient to reliably determine the SUSY parameters of non-universal models in the initial phase of LHC at $7 \mathrm{TeV}$ and with $1 \mathrm{fb}^{-1}$. This is born out by the profile likelihood contours shown in figure 6 (left). (Note that the likelihood contours for the fit without rates clearly indicate that the fit does not converge properly, even though the area enclosed by the contours is comparably small.) Adding rates, however, we obtain a reasonable fit of the larger parameter space including $M_{1}, M_{2}$ and $M_{3}$, as shown in table 3 and figure 6 . We find an accuracy of about $25 \%$ on $M_{1}, M_{2}$ and $5 \%$ on $M_{3}$ with $20 \%$ rate uncertainty, see table 3 . The high accuracy on $M_{3}$ is a result of the sensitivity of the production cross sections to the gluino mass, cf. figure 2. If we assume rates to be measured with $100 \%$ uncertainty, or if we had to rely on LO cross sections only, the accuracy on $M_{1}, M_{2}$ and $M_{3}$ is reduced to $50 \%$ and $20 \%$, respectively.

Going from $1 \mathrm{fb}^{-1}$ at $7 \mathrm{TeV}$ to $1 \mathrm{fb}^{-1}$ at $14 \mathrm{TeV}$ leads to a significant reduction of uncertainties. With rates and the observables of groups I and II included, we obtain a 


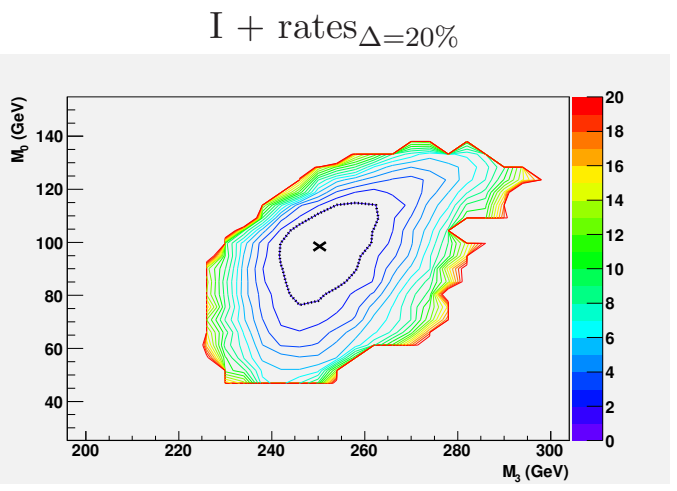

\section{$\mathrm{I}+\mathrm{II}$, rates}

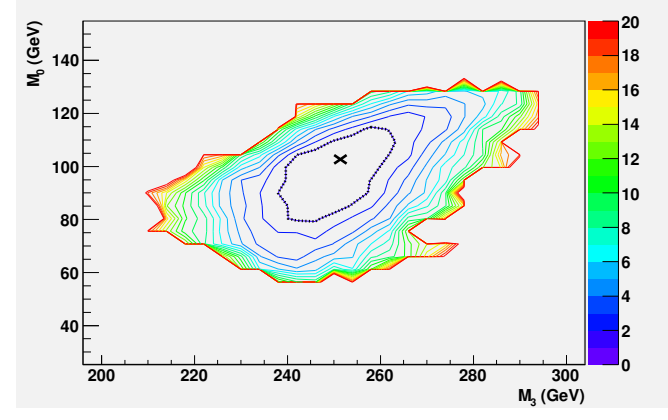

$\mathrm{I}+\mathrm{II}+\operatorname{rates}_{\Delta=20 \%}$

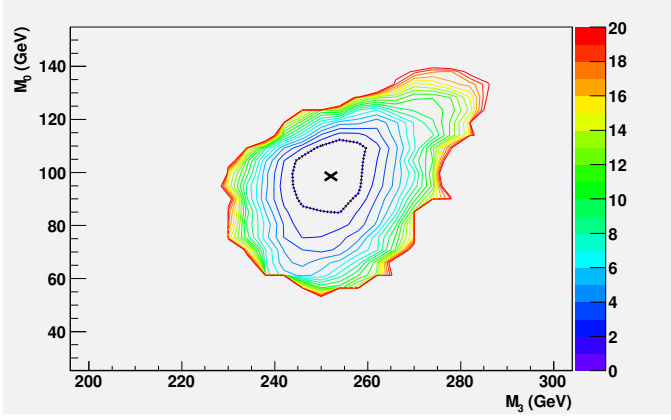

Figure 7. $\Delta \chi^{2}$ contours showing $M_{0}$ against $M_{3}$ for $14 \mathrm{TeV} / 1 \mathrm{fb}^{-1}$ data. Fits are based on the four standard edges of group I with rates (upper right), and on the observables of groups I and II with (lower right) and without rates (lower left).

good fit of the non-universal model, with errors in the range of $10 \%$ for $M_{0}, M_{1}$ and $M_{2}$, and about $3 \%$ for $M_{3}$. The fit to $\tan \beta$ has an accuracy of $50 \%$, compared to $30 \%$ for the case of universal mSUGRA fits. With $10 \mathrm{fb}^{-1}$ at $14 \mathrm{TeV}$, finally, one can determine $M_{3}$ with an error of less then $2 \%$, while the uncertainties on $M_{0}, M_{1}$ and $M_{2}$ are in the range of $5 \%$. Considering the observables of groups I and II together with event rates is sufficient to arrive at the final LHC accuracy for the GUT-scale gaugino mass parameters $M_{1}, M_{2}$ and $M_{3}$, see table 3 and figure 8.

\section{Conclusions and outlook}

Cross sections and branching ratios provide important information on the Lagrangian parameters of $\mathrm{TeV}$-scale supersymmetry. We have presented a new method to include event rates, i.e. cross sections $\times$ branching ratios $\times$ cut acceptances, into global fits of SUSY parameters at the LHC. While we expect event rates to be particularly important for SUSY scenarios that are not well constrained by the measurement of the standard kinematic edges in cascade decays, we have demonstrated that cross sections and branching ratios also add important information to fits of SPS1a-type minimal supergravity models. In particular, we find that event rates are crucial for a reliable determination of the mSUGRA 


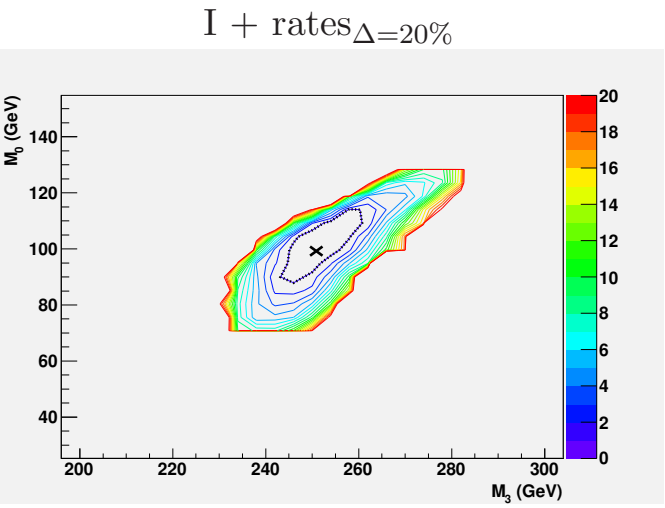

I + II, rates

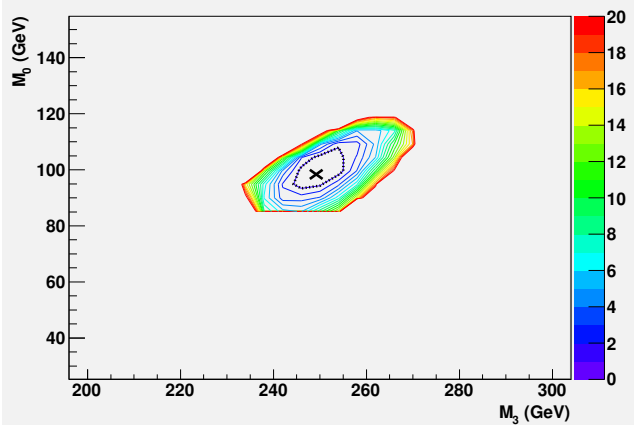

I + II + III, rates

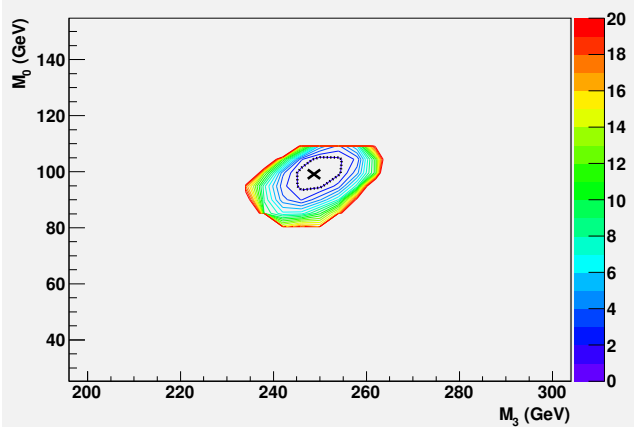

$\mathrm{I}+\mathrm{II}+\operatorname{rates}_{\Delta=20 \%}$

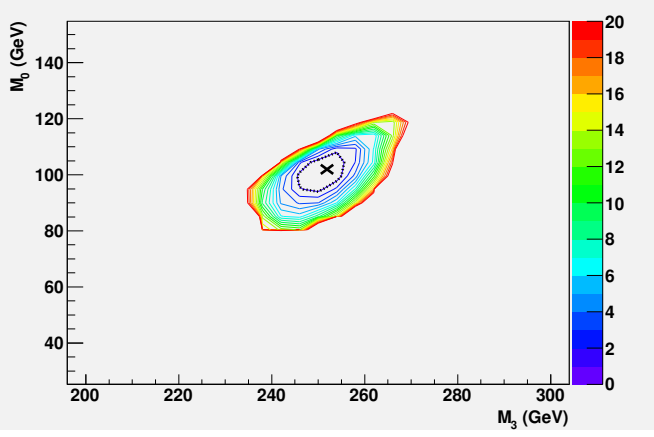

I + II + III + rates $_{\Delta=20 \%}$

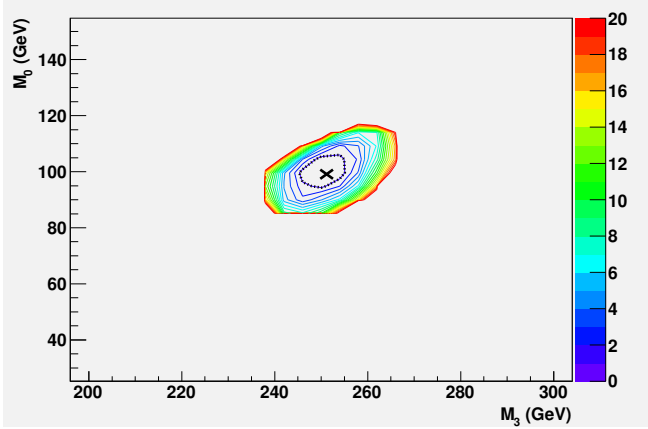

Figure 8. $\Delta \chi^{2}$ contours showing $M_{0}$ against $M_{3}$ for $14 \mathrm{TeV} / 10 \mathrm{fb}^{-1}$ data. Fits are based on the four standard edges of group I with rates (upper right), on the observables of groups I and II with (middle right) and without rates (middle left), and on observables of groups I, II, and III with (lower right) and without rates (lower left).

parameters in the initial phase of LHC data taking at $7 \mathrm{TeV}$ collision energy with $1 \mathrm{fb}^{-1}$ integrated luminosity. We have also studied a more general class of models where we allow for individual, in general non-universal, gaugino masses $M_{1}, M_{2}$ and $M_{3}$ as fit parameters instead of a common mass $M_{1 / 2}$. The parameter determination of such models improves significantly when rates are included.

The purpose of this paper is to establish a new method for including event rates into global SUSY parameter fits at the LHC and to quantitatively study their impact using 
SPS1a as a case study. Our numerical results are based on Fittino, and the calculation of event rates will be included in the next release of the Fittino program package. Note, however, that the method is general and can also be used with other fitting codes. There are many possible studies that could follow from the method and the results presented here. We shall, for example, extend the range of applicability of our cut acceptance calculation by including three-body decay modes and decays into on-shell $Z$ and Higgs bosons. Moreover, the estimated uncertainty on the acceptance should be verified by a detector simulation. So far, we have focused on LHC data alone, but shall address the interplay of LHC results with low-energy observables, other collider data and dark matter constraints in future analyses (cf. $[13,16,66-70])$. We have seen that event rates are particularly important to analyze more general models, such as models with non-universal gaugino masses. We thus plan a more comprehensive study of the determination of the gaugino mass pattern from LHC data, which may play a crucial role to distinguish models of supersymmetry breaking.

\section{Acknowledgments}

We are particularly grateful to Peter Wienemann for his invaluable help in all matters related to the technical aspects of Fittino. Thanks also to him, Philip Bechtle and Klaus Desch for many discussions on physics and fitting. We have appreciated Werner Porod's help with SPheno, and many discussions on experimental aspects of SUSY searches with Lutz Feld, Niklas Mohr, Daniel Sprenger and Matthias Edelhoff. This work has been supported in part by the Helmholtz Alliance "Physics at the Terascale", the DFG SFB/TR9 "Computational Particle Physics", the BMBF "Verbundprojekt HEP-Theorie" under contracts 05H09PDE and 05H09PAE, and the European Community's Marie-Curie Research Training Network under contract MRTN-CT-2006-035505 "Tools and Precision Calculations for Physics Discoveries at Colliders". MK would like to thank the CERN TH division for their hospitality.

Open Access. This article is distributed under the terms of the Creative Commons Attribution Noncommercial License which permits any noncommercial use, distribution, and reproduction in any medium, provided the original author(s) and source are credited.

\section{References}

[1] Y.A. Golfand and E.P. Likhtman, Extension of the algebra of Poincaré group generators and violation of p invariance, JETP Lett. 13 (1971) 323 [Pisma Zh. Eksp. Teor. Fiz. 13 (1971) 452] [SPIRES].

[2] J. Wess and B. Zumino, Supergauge transformations in four-dimensions, Nucl. Phys. B 70 (1974) 39 [SPIRES].

[3] H.P. Nilles, Supersymmetry, supergravity and particle physics, Phys. Rept. 110 (1984) 1 [SPIRES].

[4] H.E. Haber and G.L. Kane, The search for supersymmetry: probing physics beyond the standard model, Phys. Rept. 117 (1985) 75 [SPIRES].

[5] J.R. Ellis, J.S. Hagelin, D.V. Nanopoulos, K.A. Olive and M. Srednicki, Supersymmetric relics from the Big Bang, Nucl. Phys. B 238 (1984) 453 [SPIRES]. 
[6] G. Jungman, M. Kamionkowski and K. Griest, Supersymmetric dark matter, Phys. Rept. 267 (1996) 195 [hep-ph/9506380] [SPIRES].

[7] I. Hinchliffe, F.E. Paige, M.D. Shapiro, J. Soderqvist and W. Yao, Precision SUSY measurements at CERN LHC, Phys. Rev. D 55 (1997) 5520 [hep-ph/9610544] [SPIRES].

[8] B.C. Allanach, C.G. Lester, M.A. Parker and B.R. Webber, Measuring sparticle masses in non-universal string inspired models at the LHC, JHEP 09 (2000) 004 [hep-ph/0007009] [SPIRES].

[9] B.K. Gjelsten, D.J. Miller and P. Osland, Measurement of SUSY masses via cascade decays for SPS 1a, JHEP 12 (2004) 003 [hep-ph/0410303] [SPIRES].

[10] LHC/LC Study Group collaboration, G. Weiglein et al., Physics interplay of the LHC and the ILC, Phys. Rept. 426 (2006) 47 [hep-ph/0410364] [SPIRES].

[11] C.G. Lester, M.A. Parker and M.J. White, Determining SUSY model parameters and masses at the LHC using cross-sections, kinematic edges and other observables, JHEP 01 (2006) 080 [hep-ph/0508143] [SPIRES].

[12] P. Bechtle, K. Desch, W. Porod and P. Wienemann, Determination of MSSM parameters from LHC and ILC observables in a global fit, Eur. Phys. J. C 46 (2006) 533 [hep-ph/0511006] [SPIRES].

[13] R. Lafaye, T. Plehn, M. Rauch and D. Zerwas, Measuring supersymmetry, Eur. Phys. J. C 54 (2008) 617 [arXiv:0709.3985] [SPIRES].

[14] E. Turlay, The MSSM with decoupled scalars at the LHC, arXiv:0805.2272 [SPIRES].

[15] L. Roszkowski, R. Ruiz de Austri and R. Trotta, Efficient reconstruction of CMSSM parameters from LHC data - A case study, arXiv:0907.0594 [SPIRES].

[16] P. Bechtle, K. Desch, M. Uhlenbrock and P. Wienemann, Constraining SUSY models with Fittino using measurements before, with and beyond the LHC, Eur. Phys. J. C 66 (2010) 215 [arXiv: 0907.2589] [SPIRES].

[17] B.C. Allanach, SUSY predictions and SUSY tools at the LHC, Eur. Phys. J. C 59 (2009) 427 [arXiv:0805.2088] [SPIRES].

[18] H. Baer, Computational tools for supersymmetry calculations, arXiv:0912.3270 [SPIRES].

[19] P. Bechtle, K. Desch and P. Wienemann, Fittino, a program for determining MSSM parameters from collider observables using an iterative method, Comput. Phys. Commun. 174 (2006) 47 [hep-ph/0412012] [SPIRES].

[20] P. Bechtle, K. Desch and P. Wienemann, private communication.

[21] C.G. Lester, M.A. Parker and M.J. White, Three body kinematic endpoints in SUSY models with non-universal Higgs masses, JHEP 10 (2007) 051 [hep-ph/0609298] [SPIRES].

[22] H. Baer, V. Barger, G. Shaughnessy, H. Summy and L.-t. Wang, Precision gluino mass at the LHC in SUSY models with decoupled scalars, Phys. Rev. D 75 (2007) 095010 [hep-ph/0703289] [SPIRES].

[23] K. Choi and H.P. Nilles, The gaugino code, JHEP 04 (2007) 006 [hep-ph/0702146] [SPIRES].

[24] T. Appelquist, H.-C. Cheng and B.A. Dobrescu, Bounds on universal extra dimensions, Phys. Rev. D 64 (2001) 035002 [hep-ph/0012100] [SPIRES].

[25] H.-C. Cheng, K.T. Matchev and M. Schmaltz, Bosonic supersymmetry? Getting fooled at the CERN LHC, Phys. Rev. D 66 (2002) 056006 [hep-ph/0205314] [SPIRES]. 
[26] J.M. Smillie and B.R. Webber, Distinguishing spins in supersymmetric and universal extra dimension models at the Large Hadron Collider, JHEP 10 (2005) 069 [hep-ph/0507170] [SPIRES].

[27] G.L. Kane, A.A. Petrov, J. Shao and L.-T. Wang, Initial determination of the spins of the gluino and squarks at LHC, J. Phys. G 37 (2010) 045004 [arXiv:0805.1397] [SPIRES].

[28] J. Hubisz, J. Lykken, M. Pierini and M. Spiropulu, Missing energy look-alikes with $100 p b^{-1}$ at the LHC, Phys. Rev. D 78 (2008) 075008 [arXiv:0805.2398] [SPIRES].

[29] B.C. Allanach et al., The Snowmass points and slopes: benchmarks for SUSY searches, in the proceedings of the APS/DPF/DPB Summer Study on the Future of Particle Physics, June 30-July 21, Snowmass U.S.A. (2001), Eur. Phys. J. C 25 (2002) 113 [hep-ph/0202233] [SPIRES].

[30] W. Porod, SPheno, a program for calculating supersymmetric spectra, SUSY particle decays and SUSY particle production at $e^{+} e^{-}$colliders, Comput. Phys. Commun. 153 (2003) 275 [hep-ph/0301101] [SPIRES].

[31] W. Beenakker, R. Hopker, M. Spira and P.M. Zerwas, Squark production at the Tevatron, Phys. Rev. Lett. 74 (1995) 2905 [hep-ph/9412272] [SPIRES].

[32] W. Beenakker, R. Hopker, M. Spira and P.M. Zerwas, Gluino pair production at the Tevatron, Z. Phys. C 69 (1995) 163 [hep-ph/9505416] [SPIRES].

[33] W. Beenakker, R. Hopker, M. Spira and P.M. Zerwas, Squark and gluino production at hadron colliders, Nucl. Phys. B 492 (1997) 51 [hep-ph/9610490] [SPIRES].

[34] W. Beenakker, M. Krämer, T. Plehn, M. Spira and P.M. Zerwas, Stop production at hadron colliders, Nucl. Phys. B 515 (1998) 3 [hep-ph/9710451] [SPIRES].

[35] A. Kulesza and L. Motyka, Threshold resummation for squark-antisquark and gluino-pair production at the LHC, Phys. Rev. Lett. 102 (2009) 111802 [arXiv:0807.2405] [SPIRES].

[36] U. Langenfeld and S.-O. Moch, Higher-order soft corrections to squark hadro-production, Phys. Lett. B 675 (2009) 210 [arXiv:0901.0802] [SPIRES].

[37] A. Kulesza and L. Motyka, Soft gluon resummation for the production of gluino-gluino and squark-antisquark pairs at the LHC, Phys. Rev. D 80 (2009) 095004 [arXiv:0905.4749] [SPIRES].

[38] M. Beneke, P. Falgari and C. Schwinn, Soft radiation in heavy-particle pair production: all-order colour structure and two-loop anomalous dimension, Nucl. Phys. B $\mathbf{8 2 8}$ (2010) 69 [arXiv: 0907.1443] [SPIRES].

[39] W. Beenakker et al., Soft-gluon resummation for squark and gluino hadroproduction, JHEP 12 (2009) 041 [arXiv: 0909.4418] [SPIRES].

[40] G. Bozzi, B. Fuks and M. Klasen, Non-diagonal and mixed squark production at hadron colliders, Phys. Rev. D 72 (2005) 035016 [hep-ph/0507073] [SPIRES].

[41] A.T. Alan, K. Cankocak and D.A. Demir, Squark pair production in the MSSM with explicit CP-violation, Phys. Rev. D 75 (2007) 095002 [Erratum ibid. D 76 (2007) 119903] [hep-ph/0702289] [SPIRES].

[42] S. Bornhauser, M. Drees, H.K. Dreiner and J.S. Kim, Electroweak contributions to squark pair production at the LHC, Phys. Rev. D 76 (2007) 095020 [arXiv:0709.2544] [SPIRES].

[43] W. Hollik, M. Kollar and M.K. Trenkel, Hadronic production of top-squark pairs with electroweak NLO contributions, JHEP 02 (2008) 018 [arXiv: 0712.0287] [SPIRES]. 
[44] W. Hollik and E. Mirabella, Squark anti-squark pair production at the LHC: the electroweak contribution, JHEP 12 (2008) 087 [arXiv:0806.1433] [SPIRES].

[45] W. Hollik, E. Mirabella and M.K. Trenkel, Electroweak contributions to squark-gluino production at the LHC, JHEP 02 (2009) 002 [arXiv: 0810.1044] [SPIRES].

[46] E. Mirabella, NLO electroweak contributions to gluino pair production at hadron colliders, JHEP 12 (2009) 012 [arXiv:0908.3318] [SPIRES].

[47] W. Beenakker, R. Hopker and M. Spira, PROSPINO: a program for the PROduction of Supersymmetric Particles In Next-to-leading Order QCD, hep-ph/9611232 [SPIRES] [http://www.thphys.uni-heidelberg.de/ ${ }^{\sim}$ plehn/prospino/] [http://people.web.psi.ch/spira/prospino/].

[48] A.D. Martin, W.J. Stirling, R.S. Thorne and G. Watt, Parton distributions for the LHC, Eur. Phys. J. C 63 (2009) 189 [arXiv:0901.0002] [SPIRES].

[49] P.M. Nadolsky et al., Implications of CTEQ global analysis for collider observables, Phys. Rev. D 78 (2008) 013004 [arXiv:0802.0007] [SPIRES].

[50] R.D. Ball et al., A first unbiased global NLO determination of parton distributions and their uncertainties, arXiv: 1002.4407 [SPIRES].

[51] D. Berdine, N. Kauer and D. Rainwater, Breakdown of the narrow width approximation for new physics, Phys. Rev. Lett. 99 (2007) 111601 [hep-ph/0703058] [SPIRES].

[52] J.M. Lindert, SUSY parameter determination at the LHC, Diploma thesis, RWTH Aachen, Aachen, Germany (2010)

[53] M. Bähr et al., HERWIG++ physics and Manual, Eur. Phys. J. C 58 (2008) 639 [arXiv: 0803.0883] [SPIRES].

[54] N. Metropolis et al., Equation of state calculations by fast computing machines, J. Chem. Phys. 21 (1953) 1087 [SPIRES].

[55] The ATLAS collaboration, G. Aad et al., Expected performance of the ATLAS experiment - Detector, trigger and physics, arXiv:0901.0512 [SPIRES].

[56] CMS collaboration, G.L. Bayatian et al., CMS technical design report, volume II: physics performance, J. Phys. G 34 (2007) 995 [SPIRES].

[57] N. Mohr, Dilepton mass edge measurement in SUSY events with CMS, arXiv:0904.3408 [SPIRES].

[58] K. Roth, Rekonstruktion supersymmetrischer Zerfallskaskaden mit dem CMS-Experiment, Diploma thesis, RWTH Aachen, Aachen, Germany (2009).

[59] C.G. Lester and D.J. Summers, Measuring masses of semiinvisibly decaying particles pair produced at hadron colliders, Phys. Lett. B 463 (1999) 99 [hep-ph/9906349] [SPIRES].

[60] A. Barr, C. Lester and P. Stephens, $m_{\mathrm{T} 2}$ : the truth behind the glamour, J. Phys. G 29 (2003) 2343 [hep-ph/0304226] [SPIRES].

[61] B.C. Allanach, S. Kraml and W. Porod, Theoretical uncertainties in sparticle mass predictions from computational tools, JHEP 03 (2003) 016 [hep-ph/0302102] [SPIRES].

[62] D.M. Pierce, J.A. Bagger, K.T. Matchev and R.-j. Zhang, Precision corrections in the minimal supersymmetric standard model, Nucl. Phys. B 491 (1997) 3 [hep-ph/9606211] [SPIRES].

[63] D. Hooper and T. Plehn, Supersymmetric dark matter: how light can the LSP be?, Phys. Lett. B 562 (2003) 18 [hep-ph/0212226] [SPIRES]. 
[64] H.K. Dreiner et al., Mass bounds on a very light neutralino, Eur. Phys. J. C 62 (2009) 547 [arXiv:0901.3485] [SPIRES].

[65] H.K. Dreiner et al., Rare meson decays into very light neutralinos, Phys. Rev. D 80 (2009) 035018 [arXiv:0905.2051] [SPIRES].

[66] R. Trotta, F. Feroz, M.P. Hobson, L. Roszkowski and R. Ruiz de Austri, The impact of priors and observables on parameter inferences in the Constrained MSSM, JHEP 12 (2008) 024 [arXiv:0809.3792] [SPIRES].

[67] H. Flacher et al., Gfitter - Revisiting the global electroweak fit of the standard model and beyond, Eur. Phys. J. C 60 (2009) 543 [arXiv:0811.0009] [SPIRES].

[68] S.S. AbdusSalam, B.C. Allanach, F. Quevedo, F. Feroz and M. Hobson, Fitting the phenomenological MSSM, arXiv:0904.2548 [SPIRES].

[69] O. Buchmueller et al., Likelihood functions for supersymmetric observables in frequentist analyses of the CMSSM and NUHM1, Eur. Phys. J. C 64 (2009) 391 [arXiv:0907.5568] [SPIRES].

[70] Y. Akrami, P. Scott, J. Edsjo, J. Conrad and L. Bergstrom, A profile likelihood analysis of the constrained MSSM with genetic algorithms, JHEP 04 (2010) 057 [arXiv:0910.3950] [SPIRES]. 